nested case-control study was performed to collect individual information concerning classical CSD risk factors (weight, height, smoking status, blood pressure, blood glucose and cholesterolemia) from medical files for 76 cases of death from CSD (including 26 from IHD and 16 from CVD) and 237 controls, matched for attained age and birth date and counter-matched for cumulative radon exposure.

Results In the whole cohort, a significant association with cumulative radon exposure was observed for CVD mortality, but not for CSD or IHD mortality. In the case-control study, no significant unadjusted Odd-Ratio for cumulative radon exposure was observed for any endpoint. Analyses adjusted on CSD risk factors, for which missing data do not exceed 25\%, are ongoing.

Conclusions The issue of CSD associated to ionising radiation is crucial for radiation protection. The present study, allowing to consider individual data on major classical CSD risk factors, will contribute to improve knowledge on the effects of low dose exposure.

\section{CARBON NANOTUBE EXPOSURE ASSESSMENT FOR A STUDY ON EARLY BIOLOGICAL EFFECTS; THE CANTES STUDY}

${ }^{1}$ Eelco Kuijpers, ${ }^{2}$ Roel Vermeulen, ${ }^{1}$ Peter Tromp, 'Wouter Fransman, ${ }^{1}$ Derk Brouwer, ${ }^{3,4}$ Lode Godderis, ${ }^{2}$ Jelle Vlaanderen, ${ }^{1,2}$ Cindy Bekker, 'Anjoeka Pronk. ${ }^{1} T N O$, Zeist, The Netherlands; '2IRAS, Utrecht University, Utrecht, The Netherlands; ' ${ }^{3}$ Occupational, Environmental and Insurance Medicine, KU Leuven, Leuven, Belgium; ${ }^{4}$ External Service for Prevention and Protection at Work, Idewe, Heverlee, Belgium

10.1136/oemed-2014-102362.77

Objectives To assess personal exposure to carbon nanotubes for a study of early effect biomarkers among workers exposed to carbon nanotubes (CNTs).

Method Three major job categories were identified in the exposed factory: production, R\&D and office. For qualitative assessment personal 8-hr-TWA inhalable dust samples $(\mathrm{n}=5)$ were collected in all job categories and analysed by SEM-EDX. For quantitative assessment 8-hr TWA samples $(\mathrm{n}=30)$ were collected from the production and R\&D workers and analysed for elemental carbon, corrected for soot using SEM/EDX. Job activities were recorded during all measurements.

Results The qualitative analyses demonstrated the presence of CNTs in the personal breathing zone of production, R\&D and office workers. CNT agglomerates with particle sizes between $500 \mathrm{~nm}$ and $100 \mu \mathrm{m}$ were identified for production and $R \& D$ workers and between $500 \mathrm{~nm}$ and $5 \mu \mathrm{m}$ for office workers. No single CNTs were identified. The quantitative analyses demonstrated geometric mean (GM) inhalable CNT levels of $42.6 \mu \mathrm{g} / \mathrm{m}^{3}$ (min-max: 1.4-1186.5) and $4.6 \mu \mathrm{g} / \mathrm{m}^{3}$ (min-max: 0.2-59.5) for the production and R\&D workers, respectively.

Conclusions We identified exposure to clusters of CNT in production, R\&D and office workers of the CNT production facility. As expected GM exposure was higher for production than for R\&D workers, however considerable variability was observed. Additional quantitative measurements in office and production workers are ongoing. For the full set, analyses of within- and between-worker variability and activity as a determinant will be performed. This information will be used to assess personal exposure in a cross-sectional study of early health effects in the CANTES study.

\section{LUNG CANCER RISK AMONG MINNESOTA TACONITE MINING WORKERS}

Elizabeth Allen, Bruce Alexander, Jeffrey Mandel, Richard Maclehose, Gurumurthy Ramachandran. University of Minnesota, Minneapolis, MN, USA

\subsection{6/oemed-2014-102362.78}

Objectives The mining and processing of taconite results in exposures to non-asbestiform amphibole and non-amphibole minerals. Previous studies have shown that taconite mining workers are at an increased risk for developing lung cancer and mesothelioma and duration of employment has been shown to contribute to the risk of mesothelioma incidence. The objective of this analysis is to examine the relationship between duration of employment and lung cancer among Minnesota taconite workers.

Method Among a cohort of 44243 taconite workers, 1721 cases of lung cancer were identified and matched by five-year age interval to two controls. Total duration of employment was abstracted from individual work records.

Results Among the 5159 workers included in the analysis, 55\% worked less than one year and 15\% worked $1-5$ years, $5-15$ years and more than 15 years. The mean duration of employment among cases and controls was 6.7 and 7.2 years respectively. A conditional logistic regression analysis did not show an increased risk for development of lung cancer among those who worked $1-5$ years $(\mathrm{OR}=$ 1.2, 95\% CI: $0.99,1.38), 5-15$ years $(\mathrm{OR}=0.94,95 \%$ CI: 0.79 , $1.11)$, and more than 15 years $(\mathrm{OR}=0.90,95 \% \mathrm{CI}: 0.75,1.08)$ as compared to those who worked less than one year.

Conclusions Risk for development of lung cancer does not appear to be associated with duration of employment in the taconite industry. Future analyses will explore specific exposures to airborne particulates, including silica and non-asbestiform amphiboles, in this population.

\section{USING MACHINE LEARNING TO EFFICIENTLY USE MULTIPLE EXPERTS TO ASSIGN OCCUPATIONAL LEAD EXPOSURE ESTIMATES IN A CASE-CONTROL STUDY}

${ }^{1}$ Melissa C Friesen, ${ }^{1}$ Sarah I Locke, ${ }^{2}$ Dennis Zaebst, ${ }^{2}$ Susan Viet, ${ }^{3}$ Susan Shortreed, 'Yu-Cheng Chen, ${ }^{4}$ Dong-Hee Koh, ${ }^{5}$ Larissa Pardo, ${ }^{6}$ Kendra L Schwartz, ${ }^{7}$ Faith G Davis, ${ }^{8}$ Patricia A Stewart, ' ${ }^{1}$ Joanne S Colt, ${ }^{9}$ Mark P Purdue. ${ }^{1}$ Occupational and Environmental Epidemiology Branch, NCI, Bethesda, MD, USA; ${ }^{2}$ Westat, Rockville, MD, USA; ${ }^{3}$ Biostatistics Unit, Group Health Research Institute, Seattle, WA, USA; ${ }^{4}$ Carcinogenic Hazard Branch, National Cancer Center, Seoul, Republic of Korea; ${ }^{5}$ Emory University Rollins School of Public Health, Atlanta, GA, USA; ${ }^{6}$ Department of Family Medicine and Public Health Sciences and Barbara Ann Karmanos Institute, Wayne State University School of Medicine, Detroit, MI, USA; ' $S$ School of Public Health, University of Alberta, Edmonton, AB, Canada; ${ }^{8}$ Stewart Exposure Assessments, LLC, Arlington, VA, USA

\subsection{6/oemed-2014-102362.79}

Objectives We applied machine learning approaches to efficiently assist multiple experts to transparently estimate occupational lead exposure in a case-control study of renal cell carcinoma.

Method We used hierarchical cluster models to classify the 7154 study jobs with occupational history and job/industry questionnaires into 360 groups with similar responses. Each group was reviewed independently by two or three experts and was assigned probabilities of lead exposure $(<5 \%, \geq 5-<50 \%$, $\geq 50 \%)$ for three time periods $(<1980,1980-1994$, $\geq 1995)$. When the group's mean response pattern suggested within-group exposure variability, experts identified programmable conditions that defined the rating differences where possible or flagged the group for further review. After splitting jobs that overlapped 